\title{
ANÁLISE DA CONDIÇÃO DE SAÚDE BUCAL DE MULHERES EM SITUAÇÃO DE VIOLÊNCIA NO NORTE DO BRASIL
}

\section{ANALYSIS OF THE CONDITION OF BUCAL HEALTH OF WOMEN IN THE SITUATION OF VIOLENCE IN NORTH OF BRAZIL}

\author{
Jessica Miranda da Silva ${ }^{\mathrm{a}^{*}}$, Kelly Lene Lopes Calderaro Euclides ${ }^{\mathrm{b}^{*}}$, Izadora Virgolino do \\ Nascimento Borborem $^{\mathrm{c}^{*}}$, Paulla Iáddia Zarpellon Barbosa ${ }^{\mathrm{d}^{*}}$, Flávia Sirotheau Corrêa Pontes ${ }^{\mathrm{e}^{*}}$, \\ Liliane Silva do Nascimento ${ }^{\mathrm{f}^{*}}$
}

jessica_depalma@hotmail.com ${ }^{\mathrm{a}}$, kellycalderaro@hotmail.com ${ }^{\mathrm{b}}$, izaborborema@hotmail.comc, paulla.zarp@gmail.comd, flaviapontes@ufpa.bre, lilianenascimento2001@gmail.com ${ }^{\mathrm{f}}$

Universidade Federal do Pará

Data de recebimento do artigo: 10/10/2018

Data de aceite do artigo: 21/06/2019

\section{RESUMO}

Introdução: A violência de gênero é um problema de saúde pública que requer estratégias para o seu enfrentamento, prevenção e reparação de danos. Quando há ocorrência de violência física em mulheres, a cabeça e pescoço despontam como regiões frequentemente afetadas. Também, o sistema estomatognático sofre com agravos de ordem crônica e silenciosa. Objetivos: identificar os impactos à saúde bucal da mulher decorrente de agressões físicas e psicológicas, possibilitando a investigação desse fenômeno e ampliando o espaço da odontologia sobre os debates acerca do enfrentamento da violência. Material e métodos: Este estudo analisou a condição de saúde bucal de 120 mulheres em situação de violência atendidas na Delegacia de Atendimento Especializado em Belém do Pará/Brasil no ano de 2017 e 2018. Resultados: identificou-se as características sociodemográficas e quanto a sua saúde bucal. Os resultados mostraram que 40 (33\%) apresentavam entre 26 e 35 anos, 74 (61,67\%) se autodeclararam pardas, 50 (41,67\%) eram donas de casa/desempregadas, e) 56 (46,67\%) possuíam o ensino médio completo. A análise de correspondência indicou que o tipo de violência e as categorias relacionadas à satisfação com as condições dos seus dentes e boca, sensibilidade dentinária e índice CPO-D são dependentes, apresentando um nível de significância de $0,05(5 \%)$ e do critério Beta $(\beta)$ maior ou igual a 3 . Conclusões: Assim percebe-se que a alta prevalência de agravos bucais associada à situação de violência corrobora para a insatisfação e autoimagem feminina.

Palavras-chaves: Violência doméstica, saúde da mulher, saúde bucal

\section{ABSTRACT}

Introduction: gender violence is a public health problem that requires strategies for coping with, preventing and repairing harm. When the occurrence of physical violence in women, the head and neck appear as frequently affected regions. Also, the stomatognathic system suffers from chronic and silent diseases. Goal: identify the impacts to women's oral health due to physical and psychological aggressions, enabling the investigation of this phenomenon and expanding the space of dentistry on the debates about the confrontation of violence. Materials and methods: this study analyzed the oral health status of 120 women in situations of violence attended at the Specialized Attendance Office in Belém do Pará / Brazil in the year 2017 and 2018. Results: the sociodemographic characteristics and their oral health were identified. The results showed that 40 (33\%) were between 26 and 35 years of age, 74 (61.67\%) were self-declared, 50 (41.67\%) were housewives / unemployed, e) 56 (46.67\% had completed high school. The correspondence analysis indicates that the type of violence and the categories related to oral health regarding satisfaction with the conditions of their teeth and mouth, dentin sensitivity and CPO-D are dependent, presenting a level of significance of $0.05(5 \%)$ and of the Beta $(\beta)$ criterion greater than or equal to 3. Conclusions: thus, it is perceived that the high prevalence of oral complaints associated with the situation of violence corroborates for female dissatisfaction and self-image.

Keyword: Domestic violence, women's health, oral health 


\section{Introdução}

A violência doméstica é um processo complexo que desencadeia uma série de complicações para a saúde da mulher e de todos que estão ao seu redor, gerando demandas para os mais diversos setores da sociedade, sobretudo o setor saúde ${ }^{1,2,3}$. A percepção social da violência contra mulher é histórica, e nesse sentido, vem se transformando ao longo dos tempos em função das lutas políticas das mesmas ${ }^{3}$. A violência de gênero tem um forte componente cultural que não é facilmente superado por meio de leis e normas ${ }^{4}$.

A partir do final dos anos 80 , diversos países passaram a elaborar medidas e planos de ação para a erradicação da discriminação e das violências cometidas contra mulheres ${ }^{4}$. No Brasil, um importante avanço nesse sentido foi a promulgação da Lei 11.340/06, chamada de Lei Maria da Penha, que dispõe sobre violência doméstica e familiar cometida contra mulher. A lei foi importante no sentido de nomear as diversas formas de violência colaborando para sua identificação e visibilidade, além de garantir o reconhecimento dos direitos humanos das mulheres ${ }^{4,5}$.

Outra importante política de combate à violência cometida contra mulheres foi $o$ surgimento das Delegacias de Atendimento Especializado (DEAM's). Atualmente todos os 26 estados tem pelo menos uma delegacia especializada. Com esses espaços, muitas mulheres se sentiram mais protegidas e puderam aprender sobre seus direitos e buscar apoio para as situações vivenciadas pelas mesmas ${ }^{6}$.

Desde 1991 a violência baseada no gênero é reconhecida pela Organização Pan Americana de Saúde (OPAS) como um problema de saúde pública e tem se constituído em um fenômeno social que influencia sobremaneira o modo de viver, adoecer e morrer das mulheres ${ }^{7}$. A violência contra mulheres ocorre mais frequentemente no ambiente doméstico, sendo a maioria das agressões praticadas por pessoas com quem a vítima mantém ou manteve relacionamentos afetivos, como namorados, maridos e companheiros ${ }^{8}$. Em todas as fases da sua vida, seja na infância, na adolescência, na vida adulta ou na velhice, a mulher sofre violência doméstica em uma proporção maior que a dos homens ${ }^{8,9}$.
As consequências da violência doméstica são agravos que vão desde um empurrão leve até a sua morte ${ }^{9}$. Sendo de natureza crônica, estão associados a problemas como baixo peso, doenças sexualmente transmissíveis, depressão, insônia, problemas gastrointestinais, doenças cardíacas, hipertensão, entre outros ${ }^{9,10}$. Muitos profissionais inseridos nos serviços de saúde certamente se deparam com situações de violência doméstica que inicialmente se manifestam de modo silencioso, tanto que não são sequer percebidas ${ }^{10}$. Não raro, são resultantes situações graves de saúde frutos de sofrimento psicológico dentre as quais se destacam: dores crônicas (costas, cabeça, pernas, braços), síndrome do pânico, depressão, tentativa de suicídio e distúrbios alimentares ${ }^{10,11}$.

Complicações psicológicas resultantes da violência como dificuldade de relacionamento interpessoal e baixa autoestima, podem influenciar consideravelmente a percepção da mulher em relação à sua saúde bucal, influenciando seu autocuidado e causando problemas como cárie e doenças periodontais cuja causa verdadeira muitas vezes não é identificada pelos profissionais odontólogos ${ }^{11,12}$.

Outra grave consequência da violência doméstica são os agravos físicos, que resultam em traumas visíveis, sendo eles em maioria lesões do complexo maxilomandibular, como olhos roxos, laceração de lábios, fraturas dentais e ósseas, ausência de dentes, queimaduras no palato, gengiva e face ${ }^{12,13}$. Quando falamos das marcas produzidas no rosto feminino em decorrência de violência, entendemos que essas marcas causam implicações significativas, visto que a face detém alto valor simbólico e importância diferenciada em relação às demais partes do corpo, fazendo com que essas alterações sejam mais perturbadoras do que as ocorridas em qualquer outro sítio corporal ${ }^{14}$.

De acordo com algumas pesquisas na área de traumatologia bucomaxilofacial, a alta prevalência de lesões faciais traumáticas em homens e mulheres atendidos no sistema de saúde se deve principalmente à grande exposição e pouca proteção oferecida à região da cabeça, $\mathrm{e}$ mais especificamente ao rosto ${ }^{14}$. Alguns autores sugerem que as agressões à face podem ser uma das formas encontradas pelo agressor para reforçar o domínio e o controle sobre a mulher. 
As marcas deixadas atuariam como lembranças do poder exercido através dos efeitos posteriores à violência praticada. Assim cicatrizes, perdas dentárias, disfunções mastigatórias, bem como sequelas emocionais persistiriam como marcas ou lembretes dolorosos do abuso ${ }^{14,15}$.

$\mathrm{Na}$ prática odontológica, regularmente acolhem-se pacientes com traumas maxilofaciais de ordem aguda ou crônica, como fraturas dentais, lesões em tecidos moles e distúrbios da articulação temporomandibular, devendo ser melhor investigados como possíveis resultados de algum tipo de violência ${ }^{16}$.

Nesse contexto, e sabendo-se que estruturas do complexo maxilomandibular podem ser atingidas, o presente trabalho buscou identificar os impactos à saúde bucal da mulher decorrente de agressões físicas e psicológicas, possibilitando a investigação desse fenômeno e ampliando o espaço da odontologia sobre os debates acerca do enfrentamento da violência.

\section{Metodologia}

Trata-se de estudo transversal retrospectivo com amostra randomizada, constituída por 120 mulheres usuárias da Delegacia Especializada de Atendimento à Mulher (DEAM) em Belém do Pará, Brasil.

O Pará fica na região amazônica e é uma das 27 unidades federativas do Brasil, sendo a segunda maior delas em território. Sua capital é o município de Belém, que reúne em sua região metropolitana cerca de 2,3 milhões habitantes, sendo a região metropolitana mais populosa da região Norte ${ }^{17}$.

No ranking de violência do país, o Pará é o $10^{\circ}$ estado e Belém a $15^{\text {a }}$ capital com maior taxa de homicídios de mulheres ${ }^{18}$. Só em 2016, até o mês de agosto, 6457 casos já foram registrados. A maioria lesão corporal e ameaça contra a vida ${ }^{18}$.

No estado do Pará, a primeira delegacia especializada no atendimento às mulheres surgiu em 1987, como a primeira ferramenta governamental de enfrentamento à violência de gênero, com objetivo de atender todas as mulheres vítimas de violência do Estado ${ }^{17,18}$.
Em 2004, o governo do estado criou o Programa de Paz (PROPAZ), com o objetivo de articular, fomentar e alinhar políticas públicas voltadas para a infância, adolescência e juventude, visando a garantia dos direitos, o combate e a prevenção da violência e a disseminação da cultura de paz. As ações acontecem em parceria com os mais diversos setores da sociedade, governamentais e não governamentais ${ }^{18}$.

Em 2014, foi inaugurado o PROPAZ Mulher, que, junto com a DEAM, funcionam no mesmo prédio, oferecendo atendimento às mulheres com idade acima de 18 anos, em situação de violência doméstica, familiar e sexual, por meio de serviços essenciais de atenção em um único espaço. $\mathrm{O}$ trabalho é realizado de forma integrada entre as diversas instituições que desenvolvem políticas públicas do Estado, entre os quais a Secretaria de Estado de Saúde do Pará (SESPA), Fundação Santa Casa de Misericórdia do Pará, Centro de Perícias Científicas Renato Chaves e Polícia Civil. O grande diferencial do projeto é o apoio do Poder Judiciário. O modelo é pioneiro no Norte do país e deve garantir acolhimento interdisciplinar e qualificado, de maneira a promover a cidadania, evitar a revitimização e encorajar as mulheres paraenses a buscar apoio especializado ${ }^{18}$.

O PROPAZ Mulher oferece assistência multidisciplinar de áreas psicossocial, policial, pericial ejurídica de forma humanizada e integrada. Para garantir pronto atendimento, o espaço conta com agentes da Polícia Civil para registrar boletins de ocorrência e instaurar inquéritos 24 horas. O local também possui serviço pericial, com a realização de exames especializados e emissão de laudos para constatação de abuso sexual ou agressão física, além de serviço médico e jurídico, com orientação e monitoramento de processos. São oferecidos três níveis de atendimento: primário, secundário e terciário. $\mathrm{O}$ primário tem como foco a realização de campanhas de prevenção e sensibilização da sociedade, por meio de ações itinerantes nos municípios paraenses, oferecendo acesso à população de regiões ribeirinhas, rurais e quilombolas. Promove, também, campanhas em grandes eventos para o enfrentamento do abuso e exploração sexual, ao tráfico de mulheres para fins de trabalho escravo, exploração sexual e prostituição em regime de cárcere privado ${ }^{19}$. 
De modo a aprimorar o instrumento de coleta de dados, realizou-se estudo piloto em janeiro de 2016 com 15 mulheres, e subsidiou a construção e validação do instrumento de pesquisa utilizado. A coleta de dados foi de fevereiro de 2016 a junho de 2017, onde os critérios de inclusão foram: ser mulher, ter 18 anos de idade completos ou acima e ter realizado boletim de ocorrência ou procurado atendimento à DEAM. A coleta era realizada por quatro avaliadores devidamente treinados para a pesquisa, durante os turnos da manhã e tarde. As mulheres eram convidadas a participar voluntariamente da pesquisa. Após o aceite, eram encaminhadas para sala individual e reservada, onde eram examinadas. O exame clínico foi realizado baseado nos critérios adotados pela Pesquisa Nacional de Saúde bucal do manual do SBBrasil2010 ${ }^{19}$. A inspeção visual foi realizada sob a luz ambiente, com auxílio espelho clínico e sonda OMS esterilizadas nos padrões de biossegurança.

Para avaliar a severidade e cronicidade da cárie e sua inter-relação com a violência, escolheu-se o Índice de Dentes Permanente Cariados, Perdidos e Obturados CPO-D (1937), padronizando a arcada de 32 dentes. Estabeleceu-se intervalos dos resultados em relação ao número absoluto do CPO-D: 0 a 6, 7 a 13, 14 a 18, 19 a 24 e 25 a 32.

Os dados foram submetidos ao teste de análise de correspondência é uma técnica estatística exploratória utilizada para verificar associações ou similaridades entre variáveis qualitativas ou variáveis contínuas categorizadas ${ }^{20,21}$.

É uma técnica de interdependência, tem como objetivo a representação ótima da estrutura dos dados observados e sua principal característica é redução de dados a serem analisados pelo pesquisador com perda mínima de informações, transformando as linhas e colunas das tabelas em unidades correspondentes, o que facilita a representação conjunta dos dados, onde essa correspondência é fundamental para a construção dos gráficos ${ }^{20}$.

Há dois tipos de Análise de Correspondência, a simples que corresponde a aplicação de tabelas de contingência de dupla entrada e a múltipla que se trata de tabelas de contingência com múltiplas entradas.

Para validar a técnica da análise de correspondência é necessário seguir alguns pressupostos. Primeiramente, para a aplicação da técnica análise de correspondência, é recomendado que seja realizado o teste qui-quadrado $\left(\chi^{2}\right)$ para verificar a existência de dependência entre as variáveis em estudo. As hipóteses testadas são $\mathrm{H}_{0}$ : as variáveis são independentes e $\mathrm{H}_{1}$ : as variáveis são dependentes, a estatística do teste quiquadrado é dado por

$$
\chi^{2}=\sum_{i=1}^{l} \sum_{j=1}^{c} \frac{\left(O_{i j}-E_{j}\right)^{2}}{E_{j}}
$$

em que $O_{\mathrm{ij}}$ é a frequência observada e $E_{\mathrm{ij}}$ é a frequência esperada para a $i$-ésima linha e $j$-ésima coluna da tabela de contingência, definido por

$$
E_{i j}=\frac{(\operatorname{somad} \text { linha } 1) \times(\text { soma d coluna })}{\text { Total }}
$$

Com a rejeição da hipótese nula $\left(\mathrm{H}_{0}\right)$ no teste qui-quadrado $\left(\chi^{2}\right)$, o próximo passo consiste em calcular o critério $\beta$, para verificar a dependência entre as categorias das variáveis. Em que, as hipóteses testadas são $\mathrm{H}_{0}$ : as categorias das variáveis são independentes e $\mathrm{H}_{1}$ : as categorias das variáveis são dependentes. Se o valor $\operatorname{de} \beta \geq 3$, indica-se a rejeição da hipótese $\left(\mathrm{H}_{0}\right)$, concluindose que as categorias das variáveis são associadas entre si. $O$ cálculo do critério $\beta$ é obtido pela seguinte fórmula

$$
\beta=\frac{\chi^{2}-(l-1 \chi c-1)}{\sqrt{(l-1) c-1)}},
$$

em que $\chi^{2}$ é o valor do qui-quadrado; $l$ é o número de linhas e $c$ é o número de colunas da tabela de contingência ${ }^{21}$.

Outro importante pressuposto a ser analisado é o cálculo do percentual de inércia, referente à variação explicada por cada dimensão. Quando utilizada a análise de correspondência simples as associações são propagadas em um plano bidimensional, logo, a soma do percentual de inércia das dimensões 1 e 2 deve ser igual ou superior a $70 \%$ para que os resultados sejam válidos ${ }^{22}$. 
Para saber qual é a probabilidade de uma categoria de variável estar associada com outra é necessário calcular o coeficiente de confiança, utilizando um procedimento baseado nos resíduos no qual é definido pela diferença entre as frequências esperadas e as observadas. O resíduo padronizado é dado por

$$
Z_{r e s}=\frac{O_{j}-E_{j}}{\sqrt{E_{j}}} .
$$

em que $O_{\mathrm{ij}}$ é a frequência observada e $E_{\mathrm{ij}}$ é a frequência esperada calculada por meio da Equação (2).

Por fim, após a obtenção dos valores dos resíduos, calcula-se o coeficiente de confiança $(\gamma)$, para verificar a significância dos resíduos calculados, por meio de

$$
\gamma=\left\{\begin{array}{lll}
0 & \& & Z_{r e s} \leq 0 ; \\
1-2 \times\left[1-P\left(Z<Z_{r e s}\right],\right. & \& & 0<Z_{r e s}<3 ; \\
1 & \& & Z_{\text {res }} \geq 3,
\end{array}\right.
$$

sendo que $Z_{\text {res }}$ é uma variável aleatória com distribuição de probabilidade normal padrão. As associações entre as categorias são consideradas significativas, quando o valor do coeficiente de confiança indica probabilidades moderadamente significativas, isto é, quando ou quando o valor do coeficiente de confiança indica probabilidades fortemente significativas, isto é, quando $(\gamma) \geq$ $70,00 \%$.

A análise de correspondência será realizada com o auxílio do aplicativo Statistica, versão 6.0. Em todos os testes, fixou-se $\alpha=5 \%(p \leq 0,05)$ para rejeição da hipótese nula.

Este estudo respeitou a Resolução 466/12, envolvendo seres humanos, estabelecidas pelo Conselho Nacional de Saúde e foi aprovado pelo Comitê de Ética em Pesquisa da Universidade Federal do Pará sob o parecer de número 2.110.022.

\section{Resultados e Discussão}

Os resultados foram obtidos de uma amostra de 120 mulheres que fizeram parte da demanda espontânea atendida na Delegacia de Atendimento Especializado em Belém do Pará. Muitas mulheres não apresentavam traumas visíveis e esse não foi um critério de inclusão da amostra. Um aspecto que foi considerado foi o fato de muitas mulheres que procuraram o atendimento especializado se encontravam em um momento de fragilidade muitas vezes aparente, e foi facilmente notado no momento que adentravam nas dependências da DEAM. Numa percepção observacional não sistemática, era evidente que após um período mesmo que curto na sala de espera, havia uma mudança no semblante dessas mulheres, possivelmente ocasionado pelo acolhimento ambiental e por perceberem que existiam outras mulheres que vivenciavam a mesma situação ${ }^{23}$. Dessa forma foi de extrema importância que a equipe de pesquisa desenvolvesse a sensibilidade de perceber que a coleta dos dados não causaria nenhum desconforto para as entrevistadas, sendo a abordagem realizada após a percepção visual de que a entrevistada estava tranquila e à vontade para participar da pesquisa.

Dentre as 120 mulheres que compõe a nossa amostra, as idades das mulheres atendidas na DEAM-PA encontravam-se entre 26 e 35 anos $(33,33 \%)$, seguido pela faixa etária de 36 a 45 anos $(28,33 \%)$, conforme tabela 1 . Um estudo realizado no município de João Pessoa em 2009 com mulheres que procuraram a DEAM-PB mostrou que a idade das participantes variou entre $21 \mathrm{e}$ 45 anos $^{24}$. Um achado similar mostrou que no Rio de Janeiro em 2013 a faixa etária de maior vulnerabilidade à violência entre as mulheres estão entre 20 e 29 anos $^{24}$. Um estudo transversal realizado pelo Multi -Country Study on Women's Health and Domestic Violence Against Woman demonstraram que faixa etária mais vulnerável à violência encontra-se de 20 a 29 anos $^{24}$.

Em outros estudos que levantaram as condições de mulheres em situação de violência, por exemplo em um estudo de 2016 no Rio de Janeiro, encontrou-se em um atendimento de emergência hospitalar a predominância da faixa etária de 20-29 em 45,7\% dos casos de sua amostra ${ }^{25}$. Os 
dados divergem das amostras encontradas na DEAM de Belém, onde apenas 10\% das mulheres encontravam-se na faixa etária de 18 a 25 anos, ou seja, 12 mulheres da amostra total. Isso revela que quando os dados são coletados em serviços de atendimento médico a faixa etária diminui, pois os casos são resultantes de violência aguda, que causa lesões físicas que necessitam de tratamento imediato, que nem sempre resultarão em um processo-crime nas instâncias jurídicas.

O primeiro estudo brasileiro com base populacional sobre violência contra mulher que possibilitou a comparação com outros países foi realizado no período de 2000 a 2001. Foi realizado um inquérito domiciliar em mulheres residentes na cidade de São Paulo e outras 15 cidades da zona da mata de Pernambuco. Esse estudo demonstrou que a população negra foi a maior relatora de episódios de violência, e que à medida que diminuíam a renda e a escolaridade, maiores eram as proporções dos episódios de violência ${ }^{25}$.

Esses dados corroboram informações encontradas na amostra de Belém do Pará, onde $86,67 \%$ das mulheres em situação de violência de declararam pretas/pardas, e $46,67 \%$ possuem o ensino médio seguido por $36,67 \%$ que possuem apenas o ensino fundamental.

Outra publicação do mesmo estudo brasileiro mostrou que as maiores taxas de violência foram registradas nas situações em que as mulheres vitimadas eram empregadas domésticas, aposentadas, ou estavam desempregadas, e consequentemente apresentavam baixa renda ${ }^{19}$, ${ }^{20}$. Os dados são similares com a realidade das mulheres amazônidas onde 41,67\% encontramse desempregadas ou na condição de dona de casa e $45 \%$ possuem uma renda de 1 a 2 salários mínimos, conforme apresentado na tabela 1.

Tabela 1 - Distribuição de mulheres atendidas na DEAM entre setembro de 2016 e junho de 2017, segundo faixa etária, cor, ocupação, escolaridade e renda.

\begin{tabular}{lrr}
\hline Faixa Etária & $\mathbf{( N )}$ & $\mathbf{( \% )}$ \\
\hline $18-25$ anos & 12 & 10,00 \\
$26-35$ anos & 40 & 33,33 \\
$36-45$ anos & 34 & 28,33 \\
$46-65$ anos & 32 & 26,67 \\
Mais de 65 anos & 2 & 1,67 \\
Total & $\mathbf{1 2 0}$ & $\mathbf{1 0 0 , 0 0}$ \\
& & \\
\hline Cor $\quad$ & & 25,00 \\
\hline Preta & 30 & 61,67 \\
Parda & 74 & 13,33 \\
Branca & 16 & $\mathbf{1 0 0 , 0 0}$ \\
Total & $\mathbf{1 2 0}$ & \\
& & 41,67 \\
\hline Ocupação & & 6,67 \\
\hline Dona de casa/Desempregada & 50 & 13,33 \\
Estudante & 8 & 18,33 \\
Profissional Liberal & 16 & 15,00 \\
Mercado informal & 22 & 5,00 \\
Mercado Formal & 18 & $\mathbf{1 0 0}$ \\
Aposentada & 6 & $\mathbf{1 2 0}$ \\
Total & &
\end{tabular}




\begin{tabular}{lrr}
\hline Escolaridade & & \\
\hline Ensino Fundamental & 44 & 36,67 \\
Ensino Médio & 56 & 46,67 \\
Superior & 18 & 15,00 \\
Ignorado & 2 & 1,67 \\
Total & $\mathbf{1 2 0}$ & $\mathbf{1 0 0 , 0 0}$ \\
& & \\
\hline Renda & & \\
\hline Ignorado & 4 & 3,33 \\
Menos de 1 SM & 32 & 26,77 \\
De 1 a 2 SM & 54 & 45,00 \\
De 2 a 5 SM & $\mathbf{2 6}$ & $\mathbf{2 3 , 3 3}$ \\
Acima de 5 & $\mathbf{2}$ & $\mathbf{1 , 7 7}$ \\
Total & $\mathbf{1 2 0}$ & $\mathbf{1 0 0 , 0 0}$ \\
\hline
\end{tabular}

Fonte: Pesquisa Pibic- UFPA 2017/2018

O que podemos ver diante do quadro das condições socioeconômicas das mulheres em situação de violência atendidas na Deam-Pa, é que se tratam de mulheres adultas e capazes, mas que muitas vezes dependem financeiramente do agressor, outras vezes são submetidas a essa situação por medo, dependência afetiva, ou por se considerarem inferiores aos agressores.

Se tratando de uma pesquisa que aborda violência doméstica, os dados relacionados às características do agressor se mostram importantes para apresentação de um panorama mais abrangente sobre a temática.

Os dados coletados na Deam- Pa mostram que em 73,33\% dos casos o agressor foi o namorado, seguido por $21,67 \%$ dos casos em que o agressor foi o companheiro / marido. As opções de pai e vizinho constavam no instrumento, mas não foram citados na pesquisa como agressores. Em 5\% dos casos, os agressores não foram identificados.

Um estudo realizado na Deam de Porto Alegre em 2010 mostra que em 70\% dos casos levantados na pesquisa, o agressor era o companheiro da vítima $^{21}$. Segundo a Organização Mundial da
Saúde, uma em cada seis mulheres no mundo sofre violência doméstica, sendo que em $60,0 \%$ dos casos a violência foi perpetrado por marido ou companheiro ${ }^{25}$.

Outras informações sobre o agressor coletadas nesse estudo foram a escolaridade, onde a maior porcentagem $(38,33 \%)$ possuíam o nível fundamental, e a ocupação dos agressores, onde segundo as entrevistadas, $(38,33 \%)$ dos agressores possuía uma ocupação no mercado formal.

Os dados sobre os agressores encontrados nesse estudo são compatíveis com outros achados na literatura que mostram os parceiros ou ex- parceiros íntimos enquanto principais agressores $^{26}$. Essa consonância do predomínio do companheiro nos casos de violência mostra que o lar antes considerado um lugar seguro, passou a representar um risco para as mulheres em situação de violência.

Os dados relacionados à violência mostram que em 75\% dos casos a agressão não ocorreu pela primeira vez. 
Tabela 2 - Distribuição de mulheres atendidas na DEAM segundo as características dos agressores, frequência da agressão e meio de agressão. Belém, Pará, Brasil

\begin{tabular}{|c|c|c|}
\hline Agressor & $(\mathrm{N})$ & $(\%)$ \\
\hline Ignorado & 6 & 5,00 \\
\hline Marido/companheiro & 26 & 21,67 \\
\hline Namorado & 88 & 73,33 \\
\hline Total & 120 & 100,00 \\
\hline \multicolumn{3}{|l|}{ Escolaridade do agressor } \\
\hline Ignorado & 8 & 6,67 \\
\hline Não estudou & 8 & 6,67 \\
\hline Ensino Fundamental & 46 & 38,33 \\
\hline Ensino Médio & 44 & 36,67 \\
\hline Superior & 14 & 11,67 \\
\hline Total & 120 & 100,00 \\
\hline \multicolumn{3}{|l|}{ Ocupação do agressor } \\
\hline Ignorado & 8 & 6,67 \\
\hline Não trabalha & 14 & 11,67 \\
\hline Estudante & 8 & 6,67 \\
\hline Profissional Liberal & 14 & 11,67 \\
\hline Mercado informal & 30 & 25,00 \\
\hline Mercado Formal & 46 & 38,33 \\
\hline Total & 120 & 100,00 \\
\hline \multicolumn{3}{|c|}{ Se ocorre agressão pela primeira vez } \\
\hline $\operatorname{Sim}$ & 30 & 25,00 \\
\hline Não & 90 & 75 \\
\hline \multicolumn{3}{|l|}{ Quando ocorreu a agressão } \\
\hline Ficou em casa & 50 & 41,67 \\
\hline Procurou a DEAM & 64 & 53,33 \\
\hline Foi ao hospital ou UBS & 2 & 1,67 \\
\hline Outros & 4 & 3,33 \\
\hline Total & 120 & 100,00 \\
\hline \multicolumn{3}{|c|}{ Frequência dos episódios de violência } \\
\hline Ignorado & 28 & 23,33 \\
\hline 1 ou 2 vezes & 22 & 18,33 \\
\hline Semanalmente & 32 & 26,67 \\
\hline
\end{tabular}

Fonte: Pesquisa Pibic- UFPA 2017/2018

Quando perguntadas sobre a frequência com que sofriam violência $26,67 \%$ responderam que as agressões ocorrem semanalmente, seguido por $18,33 \%$ que disseram que a ocorrência se deu apenas uma ou duas vezes.
Os resultados acima apresentados são semelhantes a um estudo realizado em 2015 em uma delegacia de atendimento mostrou que grande parte das mulheres que faziam denuncia sofriam violência há mais de 10 anos, e que $80 \%$ das mulheres que saiam de casa devido as 
agressões, depois de um ou dois anos, voltam a morar com seus agressores ${ }^{26,27}$.

Quando perguntadas sobre o meio de agressão, $35 \%$ relataram que foi usado força corporal, e que após a agressão procuraram a delegacia de atendimento (DEAM) 53,33\%, seguido por $41,67 \%$ que disseram que ficaram em casa após agressão, e que somente dias depois foram a DEAM procurar ajuda.

É importante salientar a importância que as delegacias das mulheres possuem no acolhimento a esse público. Nesse ambiente, ela e sua família passam a dispor de atendimento psicológico e jurídico que irão ser estratégicos para a superação da violência, permitindo que elas se sintam mais fortes a autônomas ${ }^{28}$.
Em Belém a DEAM faz parte do Propaz Mulher, que é um projeto que procura articular ações de polícia, de perícia, atendimento psicossocial e outros serviços, e garante proteção diminuindo o quadro de sofrimento da assistida e seus familiares.

As perguntas relacionadas à saúde bucal das mulheres em situação de violência demonstram que muitas delas possuem queixas, e apresentam alguns agravos significativos como presença de sensibilidade dentinária, onde 55\% delas responderam sim a essa questão, e quando submetidas a análise de correspondência, apresentam importante relação entre violência e os agravos bucais.

Tabela 3 - Estatísticas Resultantes da Aplicação da Técnica de Análise de Correspondência as Variáveis: Satisfação em Relação aos Dentes/Boca; Dificuldade para Comer ou Tomar Líquidos Gelados ou Quentes e CPO-D.

\begin{tabular}{|c|c|c|c|c|c|c|}
\hline Variáveis & $\chi^{2}$ & $L$ & $C$ & $\beta$ & $\begin{array}{c}\% \\
\text { Inércia }\end{array}$ & $p$ \\
\hline $\begin{array}{l}\text { Tipo de Violência versus } \\
\text { Satisfação em Relação aos } \\
\text { dentes/boca }\end{array}$ & 51,08 & 2 & 2 & 50,08 & 100,00 & 0,000 \\
\hline $\begin{array}{l}\text { Tipo de Violência versus } \\
\text { Dificuldade para comer ou } \\
\text { tomar líquidos gelados ou } \\
\text { quentes }\end{array}$ & 30,17 & 2 & 2 & 29,17 & 100,00 & 0,000 \\
\hline $\begin{array}{l}\text { Tipo de Violência versus } \\
\text { CPO-D }\end{array}$ & 12,989 & 2 & 2 & 11,99 & 100,00 & 0,000 \\
\hline
\end{tabular}

Nota: ${ }^{2}$ - Valor do Qui-quadrado; L - Número de Categorias da Variável Linha; C - Número de Categorias da Variável Coluna; $p$ - Nível Descritivo e $\beta$ - Valor do Critério Beta.

A dor é um achado importante nesse contexto visto que ela sempre será um sinal clínico de alerta de prováveis alterações sistêmicas, embora não represente uma direta e infalível relação com problemas patológicos. O diagnóstico correto é de suma importância visto que pode ser confundida com outras condições dentárias que acusam sintomas similares ${ }^{29}$.

Numerosos fatores podem levar à exposição de túbulos dentinários, dentre eles se destacam: nível de higiene oral baixa, higiene oral inapropriada, contato oclusal como força excessiva e contato oclusal prematuro ${ }^{29}$.
A análise de correspondência mostrou que existem relações entre violência e os agravos bucais, conforme a tabela 4 .

A tabela 4 apresenta os dados onde observa-se que 'Mulheres que sofreram Violência Física estão associadas à Satisfação em Relação aos Dentes/ Boca do Tipo Nem Satisfeita/Nem Insatisfeita; à Ter Dificuldade para Comer ou Tomar Líquidos Gelados ou Quentes e ao CPO-D do Tipo 0 a 6 Dentes.

Mulheres que sofreram Violência Moral estão associadas à Satisfação em Relação aos Dentes/ 
Boca do Tipo Insatisfeita; à Ter Dificuldade para ao CPO-D do Tipo 7 a 13 Dentes (Tabela 4).

Comer ou Tomar Líquidos Gelados ou Quentes e

Tabela 4 - Estatísticas Resultantes da Aplicação da Técnica de Análise de Correspondência as Variáveis: Tipo de Violência; Satisfação em Relação aos Dentes/Boca; Dificuldade para Comer o Tomar Líquidos Gelados ou Quentes e CPO-D.

\begin{tabular}{|c|c|c|c|c|c|}
\hline & \multirow{2}{*}{ Categorias } & \multicolumn{4}{|c|}{ Tipo de Violência } \\
\hline & & Física & Moral & Patrimonial & Psicológica \\
\hline $\begin{array}{l}\text { Satisfação em } \\
\text { Relação aos } \\
\text { dentes/boca }\end{array}$ & $\begin{array}{l}\text { Insatisfeita } \\
\text { Nem } \\
\text { Satisfeita } \\
\text { Nem } \\
\text { Insatisfeita } \\
\text { Satisfeita } \\
\end{array}$ & $\begin{array}{c}-0,98(0,00) \\
4,22(100,00)^{*} \\
-1,43(0,00)\end{array}$ & $\begin{array}{c}1,13(74,10)^{*} \\
-3,80(0,00) \\
0,51(39,08)\end{array}$ & $\begin{array}{l}0,60(45,37) \\
-3,26(0,00) \\
1,59(88,91)^{*}\end{array}$ & $\begin{array}{c}-0,31(0,00) \\
0,79(56,82)^{* *} \\
0,13(10,36)\end{array}$ \\
\hline $\begin{array}{l}\text { Dificuldade } \\
\text { para comer ou } \\
\text { tomar líquidos } \\
\text { gelados ou } \\
\text { quentes }\end{array}$ & Não & $\begin{array}{c}-2,74(0,00) \\
2,92(99,65)^{*}\end{array}$ & $\begin{array}{l}2,43(98,48)^{*} \\
-2,59(0,00)\end{array}$ & $\begin{array}{l}-0,53(0,00) \\
0,57(42,97)\end{array}$ & $\begin{array}{l}0,65(48,71) \\
-0,70(0,00)\end{array}$ \\
\hline CPO-D & $\begin{array}{l}0 \text { a } 6 \\
7 \text { a } 13 \\
>13\end{array}$ & $\begin{array}{c}1,32(81,26)^{*} \\
-1,43(0,00) \\
-0,12(0,00)\end{array}$ & $\begin{array}{c}-0,47(0,00) \\
1,91(94,37)^{*} \\
-1,02(0,00)\end{array}$ & $\begin{array}{c}-0,94(0,00) \\
-0,92(0,00) \\
1,55(88,00)^{*}\end{array}$ & $\begin{array}{c}-0,30(0,00) \\
0,11(9,06) \\
0,19(15,23)\end{array}$ \\
\hline
\end{tabular}

Nota: ${ }^{*}$ Probabilidades moderadamente significativas, pois $50 \% \leq \gamma \times 100<70 \%$.

${ }^{\star}$ Probabilidades fortemente significativas, pois $\gamma \times 100 \geq 70 \%$.

Mulheres que sofreram Violência Patrimonial estão associadas à Satisfação em Relação aos Dentes/Boca do Tipo Satisfeita e ao CPO-D do Tipo $>13$ Dentes (Tabela 4).

Mulheres que sofreram Violência Psicológica estão associadas à Satisfação em Relação aos Dentes/Boca do Tipo Nem Satisfeita/Nem Insatisfeita (Tabela 4).

Os dados relacionados à saúde bucal das mulheres em situação de violência mostram que existe uma necessidade urgente de diminuição da morbidade bucal nessa população mostrando que existe uma evidente falha no processo de autocuidado dessa população.

\section{Conclusão}

Conclui-se que as mulheres em situação de violência doméstica em Belém possuem em sua grande maioria de 18 a 25 anos, são predominantemente pardas e sofrem com maior frequência a violência do tipo psicológica e moral. A média de CPO-D foi de 11,85, sendo que os valores 1 e 4 obtiveram maior frequência. Observou-se que a violência apresenta correspondência com os agravos apresentados e apontam nível de significância de 0,05 (5\%), onde problemas bucais existentes nas mulheres em situação de violência, possuem uma probabilidade fortemente significativa de estarem associados. Ressalta-se a necessidade de criação de políticas eficazes na prevenção e reabilitação integrais na assistência de saúde bucal das mulheres, incluindo assim o cirurgião dentista na rede de proteção e atendimento à violência. 


\section{Referências}

1. Dourado, S. M. \& Noronha, C. V. (2015). A face marcada: as múltiplas implicações da vitimização feminina nas relações amorosas. Ciência \& Saúde Coletiva, 20(9):29112920.

2. Castro, T. L. (2011). Lesões craniofaciais em mulheres vítimas de violência doméstica e familiar: registros do Departamento Médico Legal de Vitória (ES), entre 2004 e 2008. Dissertação de Mestrado. Unicamp. Piracicaba. São Paulo.

3. Miranda, M. P. M., de Paula C. S. \&Bordin I. A. (2010). Violência conjugal física contra a mulher na vida: prevalência e impacto imediato na saúde, trabalho e família. Revista Panamericana de Salud Publica, 27(4):300-8.

4. Silva, E. B., Padoin, S. M. M. \& Vianna, L. A. C. (2015). Violência contra a mulher e a prática assistencial na percepção dos profissionais da saúde. Texto Contexto Enfermagem, 24(1): 229-37.

5. Souza L T. Da expectativa à realidade: Aplicação das sanções na Lei Maria da Penha. 1ed.Rio de Janeiro: Lumen Juris, 2016

6. Santos C M. (2010) Da delegacia da mulher à lei Maria da Penha: Absorção / tradução de demandas feministas pelo Estado. Revista Crítica de Ciências Sociais. 89,153-170.

7. Nascimento, L. S., Hage, C. A., Nakano, A. M. S., Azevedo, P. S. B. \&Lettiere, A. (2012) Violence against Women and its Consequences to Women's Oral Health. Gênero na Amazônia, 2.

8. Silva, A. F., Lima, V. L. A., Sena, L. X., Gomes, V. R., Santos, A. C. B. \& Augusto, A. O. (2014). A versão da mídia e os danos potenciais de vida perdidos (APVP) de mulheres vítimas de violência. Revista do Laboratório de Estudo da Violência, 14.

9. Arboit J, Padoin SMM, Vieira LB, Paula CC, Costa MC, Cortes LF. (2017) Health care for women in situations of violence: discoordination of network professionals. Rev.Esc.Enferm.USP.2017;51:e03207.DOI:http://dx.doi. org/10.1590/S1980-220X2016013603207

10. Garbin C A S, Dias I A, Rovida T A S, Garbin A J I, Challenges facing health professionals in the notification of violence: mandatory implementation and followup procedures. Ciênc. saúde coletiva vol.20 n.6 Rio de Janeiro Jun. 2015 (http://dx.doi.org/10.1590/141381232015206.13442014 )

11. Carvalho L M F, Galo R, Siva R H A, Dentist and domestic violence: knowledge of professionals in the public and private sectors. Medicina (Ribeirão Preto) 2013;46(3): 297-304 (http://revista.fmrp.usp.br/)

12. Brasil. Ministério da Saúde. (2012). Prevenção e tratamento dos agravos resultantes da violência sexual contra mulheres e adolescentes - norma técnica. $3^{\mathrm{a}} \mathrm{ed}$.
13. Fernandes, M. M., Bouchardet, F. C. H., Tavares, G. S. V., Daruge Junior, E., Paranhos, L. R. (2012). Aspectos odontolegais relacionados ao dano estético nos processos julgados pelo TJ/RS. Odonto. 20(40): 7-12

14. Halpern, L. R. (2010). Orofacial injuries as markers for intimate partner violence. Oral Maxillofacial Surgery Clinic North American., 22; 239-246.

15. Instituto Brasileiro De Geografia E Estatística (IBGE). (2014). Área Territorial Oficial - Consulta Por Unidade Da Federação.

16. Instituto Brasileiro De Geografia E Estatística (IBGE). (2015). Estimativas Da População Residente No Brasil E Unidades Da Federação Com Data De Referência Em $1^{\circ}$ De Julho De 2015.

17. Luz, R. M. C. A. (2013). Disfunção temporomandibular e qualidade de vida em mulheres em situação de violência doméstica.

18. Miranda, M. P. M., de Paula C. S. \& Bordin I. A. (2010). Violência conjugal física contra a mulher na vida: prevalência e impacto imediato na saúde, trabalho e família. Revista Panamericana de Salud Publica, 27(4):300-8.

19. Garbin C A S, Queiroz A D G, Rovida T A S, Garbin A J I, Occurrence of Traumatic Dental Injury in Cases of Domestic Violence, Braz Dent J (2012) 23(1): 72 76, UNESP - Univ Estadual Paulista, Araçatuba Dental School, Araçatuba, SP, Brazil

20. Fávero L; Belfiore P; Silva F; Chan B. Análise dos Dados: modelagem multivariada para tomada de decisões. Rio de Janeiro: Elsevier, 2009.

21. Lopes H A M; Lopes I R M.; Santos A R J; Ramos E M L S; Almeida S S Espaço urbano e mobilidade das pessoas como construções sociais na BR-316 no estado do Pará. In: Almeida S S ; Araújo A R.; Ramos, E M L S (Org.). Segurança Pública: Gestão, Conflitos, Criminalidade e Tecnologia da Informação. 1.ed., Praia: Uni-CV, v. 1, p. 29-48, 2016.

22. Ramos E M L S; Almeida S S ; Araújo , A R (Orgs.). Segurança Pública: Uma abordagem Estatística e Computacional. Belém: Editora Universitária EDUFPA, v.1, p.101, 2008

23. Santi, L.N., Nakano, A. M. S. \& Lettiere, A. (2010). Percepção de mulheres em situação de violência sobre o suporte e apoio recebido em seu contexto social. Texto e Contexto Enfermagem, 19(3):417-24.

24. Santi, L. N. (2007). Estudo com mulheres vítimas de violência doméstica com lesões no complexo maxilomandibular e problemas associados. Tese de Doutorado. Escola de Enfermagem de Ribeirão Preto, USP, São Paulo.

25. Secretaria do Estado de Segurança Pública e Defesa Social do Pará (SEGUP PA). Disponível em: ( http://www.segup. pa.gov.br/ ) acesso em 20/02/2018 
26. Silva, R. F., Prado, M. M., Garcia, R. R., Daruge Júnior, E. \& Daruge, E. (2010). Atuação profissional do cirurgiãodentista diante da Lei Maria da Penha. Revista SulBrasileira de Odontologia, 7(1):110-6.

27. Schraiber L B , Barros C R S .(2017). Intimate partner violence reported by female and male users of healthcare units. Rev. Saúde Pública. 51.26 (https://doi.org/10.1590/ S1518-8787.2017051006385)

28. Oliveira MCA, Vieira ELR, Penha MRC, Melo EH, CaldasJúnior AF. Characterization of women victims of violent death in a metropolitan area of Northeast Brazil. RGO, Rev Gaúch Odontol. 2015;63(4):439-45. (https://doi. org/10.1590/1981-863720150003000102883)

29. Griebler C N, Borges J L. Violência contra a mulher: perfil dos envolvidos em boletins de ocorrência da Lei Maria da Penha. Psico. 2013;44(2):215-225.

\section{Como citar este artigo:}

Silva JM, Euclides KLLC, Borborem IVN, Barbosa PIZ, Pontes FSC, Nascimento LS. Saúde bucal em mulheres vítimas de violência. Rev. Aten. Saúde. 2019;17(60):63-74 